\title{
Correlated Roles of Temperature and Dimensionality for Multiple Exciton Generation and Electronic Structures in Quantum Dot Superlattices
}

\section{$\operatorname{AUTHOR}(\mathrm{S})$ :}

Chang, I-Ya; Kim, DaeGwi; Kim, Hyeon-Deuk

\section{CITATION:}

Chang, I-Ya ... [et al]. Correlated Roles of Temperature and Dimensionality for Multiple Exciton Generation and Electronic Structures in Quantum Dot Superlattices. The Journal of Physical Chemistry C 2019, 123(4): 2549-2556

\section{ISSUE DATE:}

2019-01-31

URL:

http://hdl.handle.net/2433/245028

\section{RIGHT:}

This document is the Accepted Manuscript version of a Published Work that appeared in final form in The Journal of Physical Chemistry C, copyright $\odot$ American Chemical Society after peer review and technical editing by the publisher To access the final edited and published work see https://doi.org/10.1021/acs.jpcc.8b10565.; The full-text file will be made open to the public on 7 January 2020 in accordance with publisher's 'Terms and Conditions for Self-Archiving'.; この論文は出版社版でありません。引用の際には出版社版をご確認ご利用ください。; This is not the published version. Please cite only the published version. 


\title{
Correlated Roles of Temperature and
}

\section{Dimensionality for the Multiple Exciton}

\section{Generation and Electronic Structures in the}

\section{Quantum Dot Superlattices}

\author{
I-Ya Chang, ${ }^{\dagger}$ DaeGwi Kim, ${ }^{\ddagger}$ and Kim Hyeon-Deuk ${ }^{*}, \dagger$ \\ Department of Chemistry, Kyoto University, Kyoto, 606-8502, Japan, and Department of \\ Applied Physics, Osaka City University, Osaka 558-8585, Japan \\ E-mail: kim@kuchem.kyoto-u.ac.jp
}

*To whom correspondence should be addressed

†Department of Chemistry, Kyoto University, Kyoto, 606-8502, Japan

${ }_{\ddagger}$ Department of Applied Physics, Osaka City University, Osaka 558-8585, Japan 


\begin{abstract}
Quantum dot superlattices (QDSLs), which are one-, two-, and three-dimensional periodic superlattices composed of QDs, induce dimensionality dependent quantum resonance among component QDs and thus represent a new type of condensed matter exhibiting novel energy, exciton and carrier dynamics. We focused on the two important parameters, the dimensionality and temperature, identifying their correlated roles to determine the electronic and photoexcited properties intrinsic to each QDSL at each dimensionality and temperature. We computationally demonstrated that the multiple exciton generation is significantly accelerated at higher temperature especially in the higher-dimensional QDSLs, indicating their great advantage especially at ambient temperature compared to an isolated zero-dimensional QD. Both of the dimensionality and temperature can be crucial and correlated parameters for independent tailoring of the properties of the QDSLs without changing size, shape and compositions of component QDs. The physical insights and advantage of the QDSLs we found here will lead to designing efficient and space-saving optoelectronic and photovoltaic devices which work at ambient temperature.
\end{abstract}




\section{Introduction}

Quantum dot superlattices (QDSLs), which are periodic superlattices composed of QDs, represent a new type of condensed matter drawing high fundamental and applied interest. Quantum confinement in individual QDs, combined with collective resonant couplings among QDs, leads to novel energy, exciton and carrier dynamics that are physically unique to QDSLs and not found in other natural materials. QDSLs have been actually intensively explored to realize efficient photovoltaic, optoelectronic and thermoelectric devices. ${ }^{1-28}$

It is remarkable that QDs can be ordered into QDSLs with the dimensionality as the one-dimensional (1D), two-dimensional (2D), and three-dimensional (3D) QDSLs, just like structures made from natural atoms. ${ }^{29,30}$ Optical, electrical, thermal and mechanical properties of such QDSLs are thus determined not only by individual QDs, but also by the dimensionality dependent resonant couplings through the interior nano space among adjacent QDs tuned by ligand engineering down to subnanometer regime. ${ }^{3,14,19,31-38}$ Thus, the dimensionality in the QDSLs should be a crucial parameter for independent tailoring of the electronic and photoexcited properties including the multiple exciton generation (MEG) through which multiple electron-hole pairs can be produced; ${ }^{39-41}$ the MEG is expected to increase solar cell efficiency by creating multiple carriers from a single photon absorption. ${ }^{42}$

Meanwhile, temperature can be another important parameter to control the physical properties of the QDSLs. The temperature dependence of the electronic and photoexcited properties of the QDSLs is one of the key factors which can determine the useful range of these new materials, so its investigation is crucial for their practical applications. Indeed, the temperature dependence of the carrier mobility and photoconductivity unveiled the different kinds of charge transports, thermally activated hopping transport and band-like transport, existing in the PbSe, ${ }^{1,4,17} \mathrm{CdSe},{ }^{1,24} \mathrm{InAs},{ }^{1,25}$ and $\mathrm{Au}^{21}$ QDSLs. The temperature-dependent Seebeck coefficient directly related to thermoelectric efficiency was also measured in the $\mathrm{PbTe}$ QDSLs. ${ }^{26}$ In the case of isolated zero-dimensional (0D) QDs, we computationally showed how the MEG rates as well as the density of states (DOS) and electron-phonon couplings 
drastically change according to the temperature, elucidating the physical mechanisms of their temperature dependence. ${ }^{43}$ Such computational approach should be distinguished from Fermi's rate theory using static Coulomb couplings at $0 \mathrm{~K} .{ }^{44-49}$

In the present paper, we focus on both of the two important parameters, the dimensionality and temperature, and their correlated roles to determine the electronic and photoexcited properties of the 1D, 2D and 3D QDSLs in comparison with the isolated 0D QD. We especially examine the QDSLs which exhibit the short-range quantum resonance among component QDs. The short-range quantum resonance, which appears only in the interior nano space smaller than $2 \mathrm{~nm}$ among component QDs of diameter smaller than $4 \mathrm{~nm}$, is not a simple overlap of wave functions between quantum well potentials ${ }^{50-54}$ but rather orbitaldependent delocalization into adjoining QDs accompanied by the orbital deformation. ${ }^{33,39,55}$ We will report the temperature dependence of not only static but also time-dependent dynamical quantities to characterize such QDSLs of each dimensionality, identifying the correlated roles of the dimensionality and temperature intrinsic to each QDSL. The insights we obtained here are crucial not only for understanding the physical mechanisms that govern photoexcited processes occurring in the QDSLs but also for designing compact and functionalized optoelectronic and photovoltaic devices like photodetectors and solar cells by controllably engineering their physical properties.

\section{Computational Methods and Material Designs}

We optimized a $\mathrm{Si}_{29} \mathrm{H}_{24}$ QD, where $\mathrm{Si}_{29}$ was cut off from a bulk Si crystal with the outerlayer Si atoms passivated by $\mathrm{H}$ atoms, with the Perdew, Burke and Ernzerhof (PBE) density functional and def2-SVP basis set at zero temperature by the Gaussian09 package. ${ }^{56}$ The QDSL was then made by periodically replicating the cubic cell in which the pre-optimized $\mathrm{Si}_{29} \mathrm{H}_{24}$ QD is located at the center position. The QDSLs thus have a cubic crystal structure while the interior nano space of approximately $0.1 \mathrm{~nm}$ among adjacent QDs is vacuum. 
We performed ab initio molecular dynamics (MD) simulation of the $\mathrm{Si}_{29} \mathrm{H}_{24}$ QDSLs as well as the 0D $\mathrm{Si}_{29} \mathrm{H}_{24}$ QD with Vienna Ab-initio Simulation Package. ${ }^{57}$ The PBE density functional and projector-augmented-wave pseudopotentials with a converged plane-wave basis of the $200 \mathrm{eV}$ cutoff energy were adopted for the density functional theory (DFT) calculations.

All the QDSLs and QD were brought up to aimed temperatures by velocity rescaling. After the careful and repeated velocity scaling, we computed 3 ps microcanonical MD trajectories on the ground electronic state by the Verlet algorithm of a 1 fs time step. The stable temperature fluctuations around the aimed temperatures were confirmed. Note that all the stable QDSLs including the 1D beads and 2D sheet which have asymmetric interactions, namely, only one-directional and two-directional interactions between $\mathrm{Si}_{29} \mathrm{H}_{24} \mathrm{QDs}$, respectively, were computationally attained without any geometry constraint even at the finite temperatures.

We finally simulated the photoexcited dynamics including the MEG based on the timedomain DFT with adiabatic Kohn-Sham (KS) bases. ${ }^{58}$ The current photoexcited dynamics involves ground, single exciton (SE) and double exciton (DE) bases, $\left|\Phi_{g}(\mathbf{r} ; \mathbf{R})\right\rangle,\left|\Phi_{S E}^{i, j}(\mathbf{r} ; \mathbf{R})\right\rangle$ and $\left|\Phi_{D E}^{i, j, k, l}(\mathbf{r} ; \mathbf{R})\right\rangle$, respectively, defined by the second quantization,

$$
\left|\Phi_{S E}^{i, j}\right\rangle=\hat{a}_{i}^{\dagger} \hat{a}_{j}\left|\Phi_{g}\right\rangle, \quad\left|\Phi_{D E}^{i, j, k, l}\right\rangle=\hat{a}_{i}^{\dagger} \hat{a}_{j} \hat{a}_{k}^{\dagger} \hat{a}_{l}\left|\Phi_{g}\right\rangle
$$

Here, an electron is generated and annihilated by the electron creation and annihilation operators, $\hat{a}_{i}^{\dagger}$ and $\hat{a}_{j}$ in the $i$ th and $j$ th adiabatic KS orbitals, respectively. Substitution of the expanded form of the total wave function,

$$
|\Psi(t)\rangle=C_{g}(t)\left|\Phi_{g}\right\rangle+\sum_{i, j} C_{S E}^{i, j}(t)\left|\Phi_{S E}^{i, j}\right\rangle+\sum_{i, j, k, l} C_{D E}^{i, j, k, l}(t)\left|\Phi_{D E}^{i, j, k, l}\right\rangle
$$

into the time-dependent Schrödinger equation leads to the equations of motion for its ex- 
pansion coefficients,

$$
\begin{aligned}
i \hbar \frac{\partial C_{X}(t)}{\partial t} & =C_{X}(t) E_{X}-i \hbar C_{g}(t) \mathbf{d}_{X ; g} \cdot \dot{\mathbf{R}}-i \hbar \sum_{i^{\prime}, j^{\prime}} C_{S E}^{i^{\prime}, j^{\prime}}(t) \mathbf{d}_{X ; S E, i^{\prime}, j^{\prime}} \cdot \dot{\mathbf{R}} \\
& -i \hbar \sum_{i^{\prime}, j^{\prime}, k^{\prime}, l^{\prime}} C_{D E}^{i^{\prime}, j^{\prime}, k^{\prime}, l^{\prime}}(t) \mathbf{d}_{X ; D E, i^{\prime}, j^{\prime}, k^{\prime}, l^{\prime}} \cdot \dot{\mathbf{R}} .
\end{aligned}
$$

A state $X$ expresses either ground, SE or DE state with the state energy $E_{X}$. Electronphonon couplings are introduced by,

$$
\mathbf{d}_{X ; Y} \cdot \dot{\mathbf{R}} \equiv\left\langle\Phi_{X}\left|\nabla_{\mathbf{R}}\right| \Phi_{Y}\right\rangle \cdot \dot{\mathbf{R}}=\left\langle\Phi_{X}\left|\frac{\partial}{\partial t}\right| \Phi_{Y}\right\rangle
$$

which cause transitions from each of the ground, SE and DE states to another state. Because the electron-phonon couplings stemmed from the dependence of the adiabatic KS orbitals on the real-time phonon dynamics $\mathbf{R}(t)$ and is proportional to the nuclear velocity $\dot{\mathbf{R}}$, transitions could never occur under the Born-Oppenheimer approximation. We performed the present photoexcited dynamics simulations including the MEG by directly solving the equations of motion, Equation (3), with the electron-phonon couplings, Equation (4), and the state energies, $E_{X}$. The time-dependent electron-phonon couplings and state energies were directly obtained from the microcanonical MD trajectories of the $\mathrm{Si}_{29} \mathrm{H}_{24}$ QDSLs and QD at the aimed temperatures. ${ }^{59-61}$ Our photoexcited simulation explicitly includes real-time phonon dynamics, and makes it possible to discuss the temperature dependence accounting for fluctuations of the state energies and electron-phonon couplings at each temperature. ${ }^{43,62}$

\section{Results and Discussions}

We graphically define the dimensionality to characterize the 3D, 2D, and 1D QDSLs as well as the 0D isolated QD in Figure 1. Each QDSL is composed of H-passivated $\mathrm{Si}$ QDs, $\mathrm{Si}_{29} \mathrm{H}_{24}$, of $1.1 \mathrm{~nm}$ diameter; silicon is a photostable, non-toxic, abundant, and technologically wellestablished material. ${ }^{63-67}$ The QDSLs can be formed by manipulating the interior nano space 


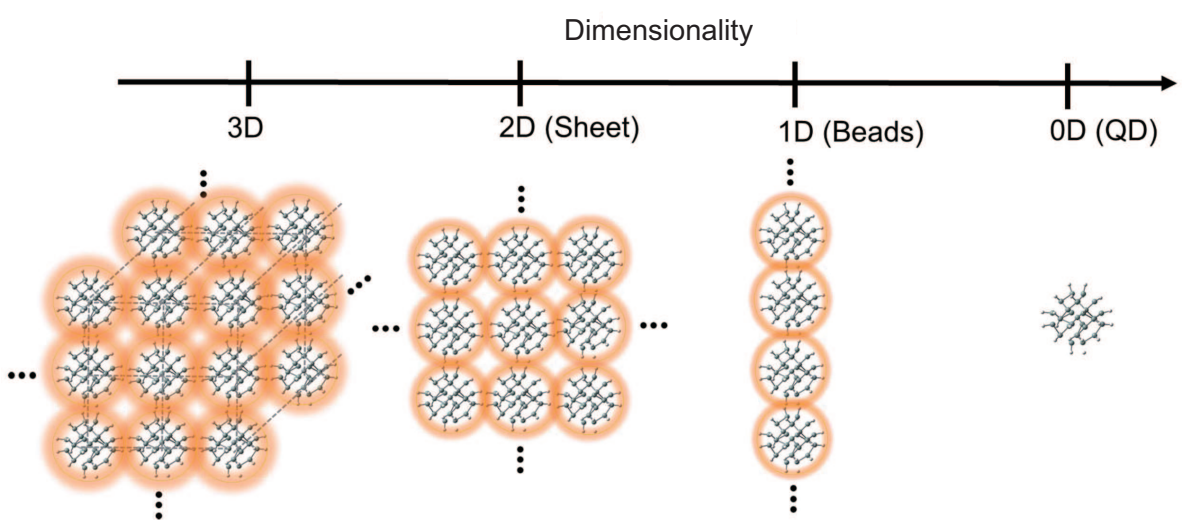

Figure 1: Graphical definition of (a) 3D, (b) 2D (sheet), and (c) 1D (beads) $\mathrm{Si}_{29} \mathrm{H}_{24}$ QDSL and (d) $0 D \mathrm{Si}_{29} \mathrm{H}_{24}$ QD. The quantum resonance induced in the QDSLs is schematically drawn with the orange rings. The quantum resonance becomes stronger in the order of the dimensionality because each QD in the higher-dimensional QDSLs has more chances to be resonant with more adjacent QDs.

between nearest-neighbor QD surfaces in each 2D layer as well as the interior nano space between adjacent $2 \mathrm{D}$ layers. ${ }^{33}$ The short-range quantum resonance among component QDs is intrinsically induced in the QDSLs with the intensity depending on the dimensionality; the higher the dimensionality is, the stronger the quantum resonance becomes because each QD in the higher-dimensional QDSLs has more chances to be resonant with adjacent QDs along more directions as demonstrated in Refs.32 and 38. We will discuss the temperature dependence of important electronic and photoexcited dynamical quantities to characterize the QDSLs focusing on the correlated role of the dimensionality.

We adopted the four temperatures, $30 \mathrm{~K}, 77 \mathrm{~K}, 100 \mathrm{~K}$, and $300 \mathrm{~K}$, to investigate the temperature dependence correlated to the dimensionality. As shown in Figure S1, the stable temperature fluctuations around each aimed temperature were successfully achieved. The stable and significant band-gap energy $\left(E_{g}\right)$ fluctuations were achieved reflecting thermal modulations of the QDSL structures at each finite temperature.(Figure S2) The averages of fluctuating $E_{g}$ are compared in Figure 2 with the multi-dependence on the temperature and dimensionality. On one hand, the stronger quantum resonance induced in the higherdimensional QDSLs directly causes the evident $E_{g}$ decrease; ${ }^{39,55} E_{g}$ decreases by more than 


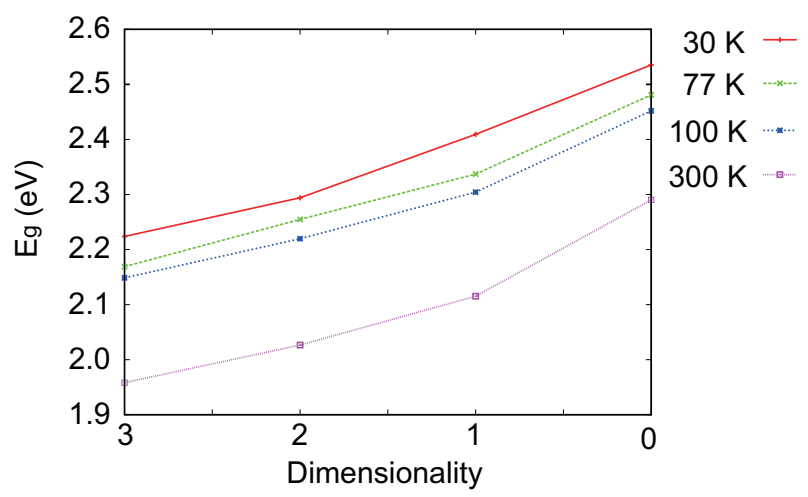

Figure 2: Average band gap energies of the $\mathrm{Si}_{29} \mathrm{H}_{24}$ QDSLs and 0D $\mathrm{Si}_{29} \mathrm{H}_{24}$ QD as a function of the temperature and dimensionality.

$0.3 \mathrm{eV}$ simply by changing the dimensionality from 0D to 3D. On the other hand, in all the QDSLs and the 0D QD, the average $E_{g}$ is higher at the lower temperature because the thermal effects of the QD expansion as well as the structural modulation is smaller at the lower temperature. Thus, the higher-dimensionality together with rising the temperature drastically decrease $E_{g} ; E_{g}$ even falls down to $1.96 \mathrm{eV}$ for the 3D QDSL at $300 \mathrm{~K}$. We also found that the changes of $E_{g}$ along the dimensionality are almost similar independent of the temperature: The dependence of the quantum resonance on the dimensionality is almost independent of the temperature. Figure 2 clearly shows the correlated role of the temperature and dimensionality for the $E_{g}$ shift and how they compensate each other; e.g. $E_{g}$ of the 0D $\mathrm{QD}$ at $300 \mathrm{~K}$ is almost similar to $E_{g}$ of the 3D QDSL at $30 \mathrm{~K}$-the 10 times difference in the temperature is compensated only by the difference in the 0D-3D dimensionality.

Figure 3 shows the DOS for SE, a pair of electron and hole, and for DE, two pairs of electrons and holes, with the dependence on the temperature and dimensionality. The SE DOS was obtained by counting the number of SE states fluctuating at each finite temperature along the 3 ps microcanonical MD trajectory. As the insert of Figure 3(a) shows, the higher temperature makes the SE DOS greatly smoother in the 3D QDSL. While a few islands of the SE DOS appear in the low-energy region at $30 \mathrm{~K}$, the islands become merged into the one peak at $300 \mathrm{~K}$ and continuously linked to the higher-energy broad peak. Such tendency 


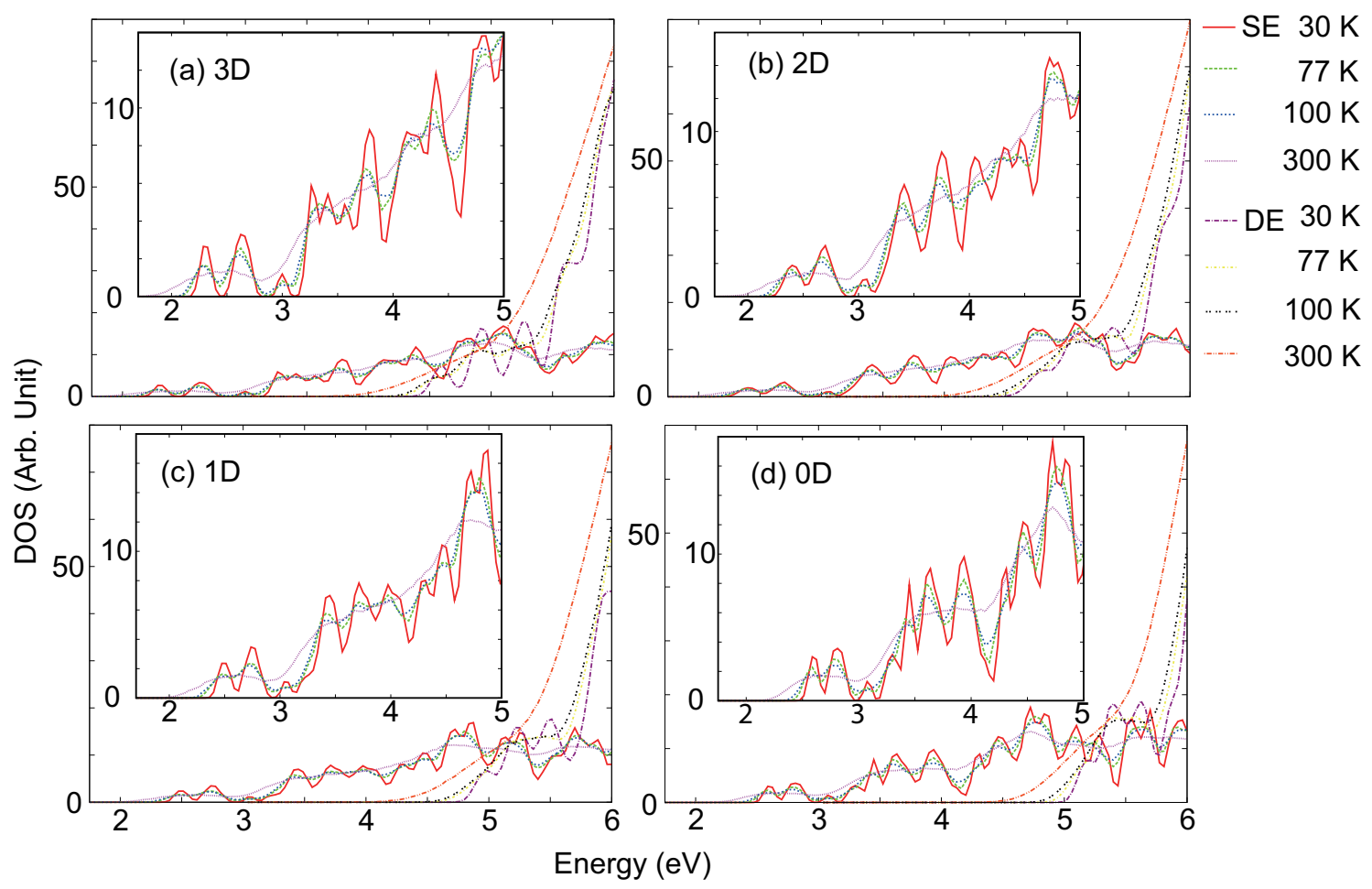

Figure 3: Temperature-dependent DOS of SE and DE states for the (a) 3D, (b) 2D, and (c) 1D $\mathrm{Si}_{29} \mathrm{H}_{24}$ QDSLs and (d) 0D $\mathrm{Si}_{29} \mathrm{H}_{24}$ QD as a function of excitation energy. The enlarged SE DOS is shown as each insert. 
can be seen also in the other QDSLs and in the 0D QD as shown in the inserts of Figure $3(b)-(d)$.

The main panels of Figure 3 show that the DE DOS starts at twice higher energy than the SE DOS, and then the DE DOS rapidly dominates the SE DOS in the higher-energy region due to the larger combination number of two electrons and two holes. The DE DOS of the QDSLs and 0D QD keeps the basic properties of the corresponding SE DOS at the same temperature; e.g. the DE DOS are smoother at the higher temperature just as the corresponding SE DOS are. The lower-energy onset of the SE DOS in the higher-dimensional QDSLs reduces the threshold energy for the MEG. Further, the ratio of the DE DOS over the SE DOS at the same excitation energy becomes larger when the SE DOS starts at the lower excitation energy, leading to the more efficient MEG ${ }^{8-10,33,34,68}$

The original valence band (VB) and conduction band (CB) DOS is provided in Figures S3 and S4, respectively. The VB DOS also significantly changes depending on the temperature regardless of the dimensionality.(Figure S3) Smoothing of the VB DOS at the higher temperature directly leads to smoothing of the SE DOS at the higher temperature shown in Figure 3. This is also the case with the CB DOS. (Figure S4) The island of the CB DOS appearing around $0 \mathrm{eV}$ contributes to the islands appearing in the SE DOS in the low-energy region.

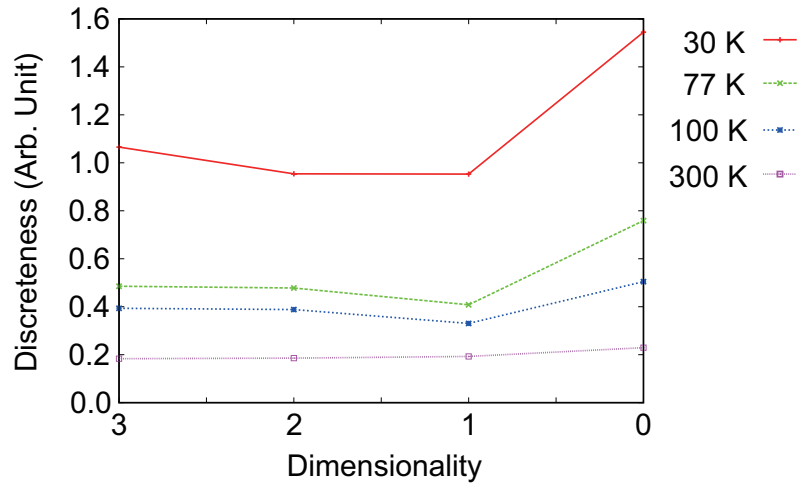

Figure 4: Discreteness of the SE DOS as a function of the temperature and dimensionality.

Figure 4 shows the discreteness of the SE DOS defined by an average of root-mean-squared 
deviation between neighboring DOS data points. Smoothing of the SE DOS is important since it contributes to the MEG acceleration. ${ }^{55}$ Especially, the smoother DE DOS, which directly stemmed from the smoother SE DOS, leads to the accelerated MEG because the MEG can occur more easily in the more continuously prepared final DE states. At $300 \mathrm{~K}$, the discreteness of the SE DOS decreases almost in the order of the dimensionality reflecting the stronger quantum resonance induced in the higher-dimensional QDSLs; the higher the dimensionality is, the smoother the DOS becomes. ${ }^{55}$ However, the lower temperature cases exhibit the qualitatively different tendency. At the lower temperature, the SE DOS of the asymmetric 1D and 2D QDSLs starts to have smaller discreteness compared to the SE DOS of the symmetric 3D QDSL and 0D QD. This is because the asymmetric orbital deformation of the $\mathrm{Si}_{29} \mathrm{H}_{24}$ QD stemmed from the asymmetric quantum resonance in the asymmetric 1D and 2D QDSLs breaks and weakens the original three-dimensionally symmetric crystalline Si-Si bonds in each $\mathrm{Si}_{29} \mathrm{H}_{24}$ QD, ${ }^{39}$ making the crystalline bonds more amorphous-like. The amorphous-like QD structure loosens the originally degenerated SE energy levels in the symmetric $\mathrm{Si}_{29} \mathrm{H}_{24}$ QD, making them more continuous. Such static and electronic effect is more enhanced at the lower temperatures where the thermal effects of the structural modulation and phonon dynamics are modest. At the higher temperature, the thermal effects become more apparent and dominate the lift of the energy degeneracy, leading to the above simple order of the discreteness with the dimensionality.

Figure S5 displays real-time DE population dynamics started from an initially excited SE state of $5.00 \mathrm{eV}$ where the ratio of the DE DOS over the SE DOS is relatively low and the MEG hardly takes place especially in the 0D QD or at the lower temperature. In addition, the $\mathrm{Si}_{29} \mathrm{H}_{24}$ QD is smaller in size than an typical experimental QD, and the ratio of the DE DOS to the SE DOS is generally lower now. ${ }^{32,69}$ Note that the current $\mathrm{Si}_{29} \mathrm{H}_{24}$ QD maintains similar Auger properties to larger QDs, and can be discussed in the same line of experimental QDs. ${ }^{55}$ The initial energy deviation among all the shown MEG simulations is only about $1.47 \%$ at maximum, which does not significantly affect the current DE population dynamics. 
We found that the significant temperature dependence of the MEG appears not only in the 0D QD but also in all the QDSLs; the population of DE states grows more rapidly as the temperature increases irrespective of the dimensionality.

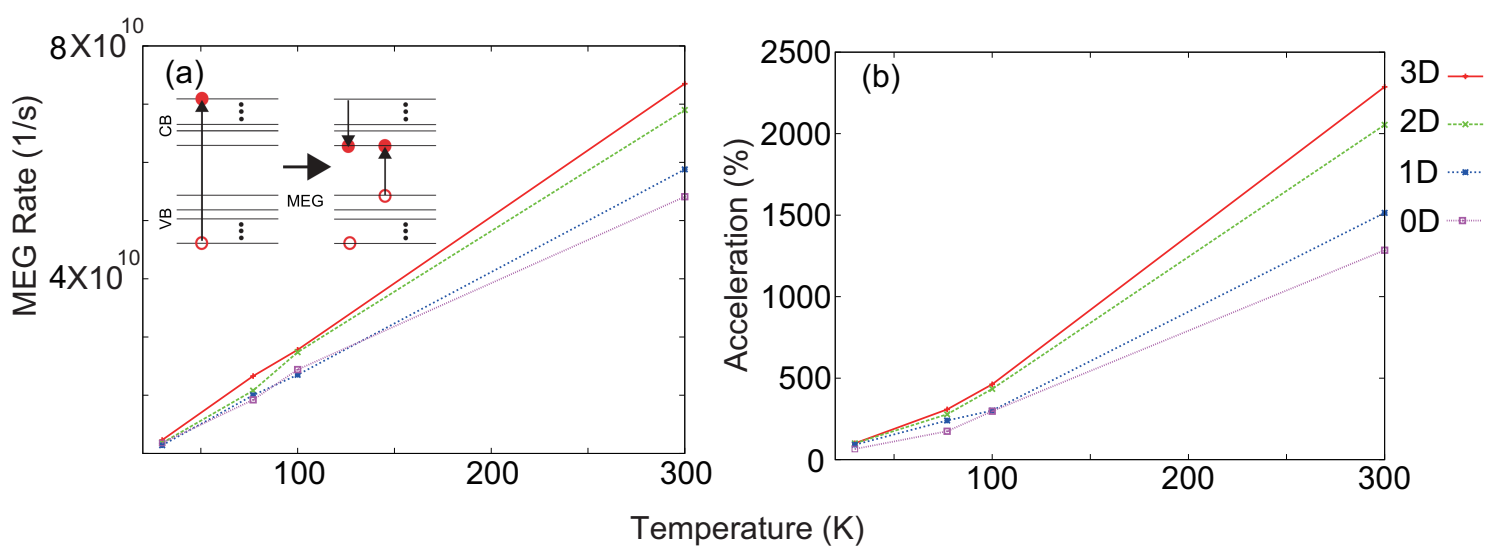

Figure 5: (a) MEG rates with the temperature and dimensionality dependence directly estimated from the real-time DE population dynamics. (b) MEG acceleration with the temperature and dimensionality dependence estimated from the actual DE population at 2 ps. The inset is a schematic figure of the MEG. Because a number of SE and DE states are involved at once in the current MEG, it is almost impossible to specifically show the SE and DE states involved in the MEG dynamics.

The MEG rates obtained by fitting the real-time DE profiles in Figure S5 are summarized in Figure 5(a). The rate rise of the MEG taking place in the 0D $\mathrm{Si}_{29} \mathrm{H}_{24}$ QD becomes gentler at the higher temperature and the gradient of the profile of the MEG rate becomes smaller with increasing the temperature. ${ }^{43}$ In contrast, the MEG rates of the QDSLs grow almost linearly with increasing the temperature and little slowing down of the rate rise appears even at the higher temperature. Indeed, although the MEG rates of the QDSLs and OD QD are almost overlapped at $30 \mathrm{~K}$, the difference among the MEG rates becomes more evident at $300 \mathrm{~K}$. The current finding means that the QDSLs have great advantage in the MEG especially at ambient temperature compared to the 0D QD. The physical origin of the different temperature dependence on the dimensionality will be discussed below. We defined the MEG acceleration as a rate of increase of the DE population at 2 ps and calculated the relative increase ratios at each dimensionality and temperature by setting the DE population 
of the $0 \mathrm{D}$ QD case at $2 \mathrm{ps}$ at $30 \mathrm{~K}$ as the origin. This MEG acceleration also demonstrates that the MEG is more enhanced in the higher-dimensional QDSLs especially at the higher temperature. (Figure 5(b)) Since the DE population grows nonlinearly, the current MEG acceleration will be further enhanced as time goes by. We conclude that the 3D QDSL is better for the MEG at ambient temperature than the lower QDSLs and 0D QD.

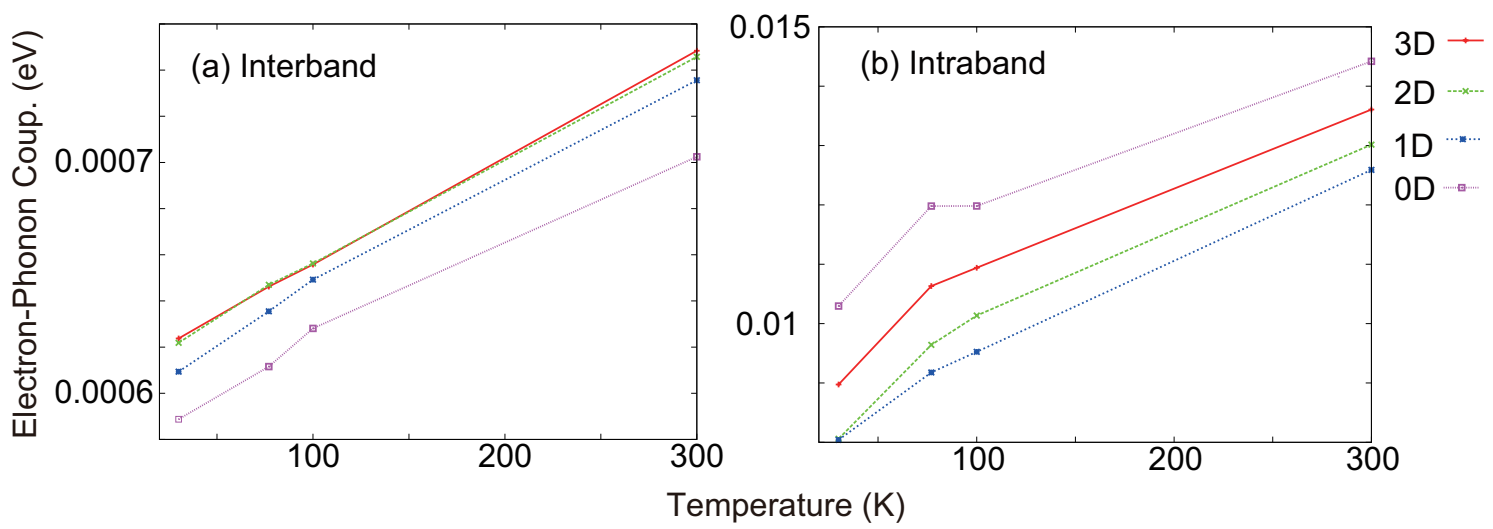

Figure 6: Root mean squares of electron-phonon couplings, Equation (4), related to (a) interband and (b) intraband carrier dynamics as a function of the temperature and dimensionality.

The electron-phonon couplings have essentially influenced photoexcited dynamics including the MEG. ${ }^{43,55,70}$ The interband electron-phonon couplings, which cause carrier transitions across the band gap, are compared in Figure 6(a). The interband electron-phonon couplings are almost linearly proportional to the temperature independent of the dimensionality, which cannot rationalize the different temperature dependence of the MEG discussed in Figure 5. The dimensionality dependence of the interband electron-phonon couplings is kept at any temperature; the higher the dimensionality is, the stronger the interband electron-phonon couplings are at all the temperatures. The 0D QD has the weakest interband electron-phonon coupling because the interband orbital overlap of two states $\left\langle\Phi_{X}\left|\nabla_{\mathbf{R}}\right| \Phi_{Y}\right\rangle$ appearing in the electron-phonon coupling, Equation (4), becomes smallest due to the largest band gap. In contrast, the orbital deformation caused by the quantum resonance in the QDSLs matches a parity of the orbital angular momenta to increase the overlap integral $\left\langle\Phi_{X}\left|\nabla_{\mathbf{R}}\right| \Phi_{Y}\right\rangle$, leading 
to their larger interband electron-phonon couplings.

The intraband electron-phonon couplings shown in Figure 6(b), which induce carrier transition without crossing the band gap, exhibit the qualitatively different behaviors from the interband electron-phonon couplings. First, the intensity of intraband electron-phonon couplings is in the order of $0 \mathrm{D}, 3 \mathrm{D}, 2 \mathrm{D}$ and $1 \mathrm{D}$ at any temperature. This anomalous dimensionality dependence of the intraband electron-phonon couplings can be attributed to partial collapse of the Si-Si crystalline phonons taking place in the asymmetric QDSLs: The asymmetric orbital deformation of each $\mathrm{Si}_{29} \mathrm{H}_{24}$ QD achieved by the asymmetric interior nano space included in the asymmetric 1D and 2D QDSLs partially breaks and weakens their Si-Si crystalline phonon modes regardless of the extent of thermal fluctuations at any temperature. In the cases of the symmetric OD QD and 3D QDSL, the Si-Si crystalline phonon modes are less affected and the resulting intraband electron-phonon couplings have the larger intensity at any temperature; the 0D QD has further freer interior nano space than the 3D QDSL, leading to its largest intraband electron-phonon coupling. Note that the low-frequency Si-Si crystalline phonon modes are directly related to low-energy intraband transitions of carriers and thus essentially determine the current intraband electron-phonon couplings. Second, although all the intraband electron-phonon couplings increase with the higher temperature, the rise becomes gentler at the higher temperature irrespective of the dimensionality. In other words, the intraband electron-phonon couplings rapidly decrease at the temperature lower than $77 \mathrm{~K}$. The drastic decrease at the lower temperature can be explained by the fact that the low-frequency Si-Si crystalline phonon modes start to freeze at the extremely lower temperature without regard for the dimensionality and, in addition, their number included in each $\mathrm{Si}_{29} \mathrm{H}_{24}$ QD is quite huge.

The current characteristic temperature dependence of the intraband electron-phonon couplings explains the logarithmic temperature dependence of the MEG rates found in the isolated $\mathrm{Si}_{29} \mathrm{H}_{24}$ QD before. ${ }^{43}$ The intraband electron-phonon coupling played an important role in diffusion of initial SE states, i.e., in preparing more pathways to final DE states 
through the MEG, and essentially determined the MEG acceleration in the case of the 0D QD. However, this is not the case with the higher-dimensional QDSLs now: Although their intraband electron-phonon couplings have the similar temperature dependence to the OD QD case, the increase of the MEG rates at the higher temperature is larger in the higher-dimensional QDSLs. (See Figure 5) We can rationalize the qualitatively different temperature dependence between the intraband electron-phonon couplings whose intensity increase slows down at the higher temperature and the linearly increasing MEG rates of the higher-dimensional QDSLs by the stronger quantum resonance and the resulting smaller $E_{g}$. (See Figure 2) The original MEG deceleration at the higher temperature, which should have been caused by the decrease in the intensity rise of the intraband electron-phonon couplings, is compensated by the $E_{g}$ decrease occurring in the higher-dimensional QDSLs at the higher temperature. It means that the MEG is determined not only by the dynamical electronphonon coupling but also by the static quantum resonance in the QDSLs especially at the higher temperature. Actually, the QDSLs, which possess the weaker intraband electronphonon couplings than the OD QD, exhibit the faster MEG than the 0D QD as shown in Figure 5. The quantum resonance of the QDSLs stably supports the MEG even under the larger thermal fluctuations at the higher temperature.

In the case of the $0 \mathrm{D} \mathrm{QD}$, the interband electron-phonon coupling is weakest while the intraband electron-phonon coupling is strongest compared to the QDSLs. Therefore, the latter electron-phonon coupling affects the MEG most significantly in the 0D QD. In addition, the $E_{g}$ decrease by the quantum resonance cannot be expected in the 0D QD. All of these facts result in its slowing down of the MEG rate at the higher temperature mainly following the temperature dependence of the intraband electron-phonon coupling. 


\section{Concluding Remarks}

We demonstrated the correlated roles of the temperature and the dimensionality to determine the electronic structures and photoexcited dynamics appearing in the QDSLs by revealing how the temperature dependence changes with the dimensionality and vice versa. The revealed correlated roles of the dimensionality and the temperature are intrinsic to the QDSLs. We conclude that the rate rise of the MEG does not slow down even at the high temperature in the 3D QDSL, suggesting that the 3D QDSL can be a strong candidate for an efficient optoelectronic and photovoltaic device to use at ambient temperature. It should be emphasized that the efficient MEG at the high temperature can be achieved by manipulating only the dimensionality of the QDSLs not by changing size, shape, and compositions of component QDs. In addition, effective charge separation, which can outpace the Auger recombination causing non-radiative recombination loss, will take place by the qualitatively different delocalization of $\mathrm{CB}$ and VB orbitals in the QDSLs. ${ }^{39}$ Significant suppression of the Auger recombination as well as the hot carrier extraction thus can be expected in the QDSLs, leading to the further higher MEG efficiency.

Passivating QDs by various kinds of ligands, especially by charged ligands, can make the quantum resonance stronger and thus will further enhance the present advantages of the QDSLs. The physical insights and advantages of the QDSLs we found here will lead to designing efficient and space-saving optoelectronic and photovoltaic devices which work at ambient temperature.

Supporting Information. Real-time temperatures and band gap energies, CB and VB DOS, and real-time DE population. 


\section{Acknowledgments}

KHD thanks the financial supports from JST (PRESTO), Japan Association for Chemical Innovation (JACI), Cybermedia Center of Osaka University, Toyota Mobility Foundation, and Grant-in-Aids for Scientific Research from Japan Society for the Promotion of Science (KAKENHI), Grant No. 15K05386. D.K. acknowledges the financial support by KAKENHI Grant No. 24560015.

\section{References}

(1) Kagan, C. R.; Murray, C. B. Charge Transport in Strongly Coupled Quantum Dot Solids. Nat. Nanotech. 2015, 10, 1013-1026.

(2) Calvo, M. E.; Hidalgo, N.; Schierholz, R.; Kovács, A.; Fernández, A.; Bellino, M. G.; Soler-Illia, G. J. A. A.; Miguez, H. Full Solution Processed Mesostructured Optical Resonators Integrating Colloidal Semiconductor Quantum Dots. Nanoscale 2015, 7, $16583-16589$.

(3) Baranov, A. V.; Ushakova, E. V.; Golubkov, V. V.; Litvin, A. P.; Parfenov, P. S.; Fedorov, A. V.; Berwick, K. Self-Organization of Colloidal PbS Quantum Dots into Highly Ordered Superlattices. Langmuir 2015, 31, 506-513.

(4) Talgorn, E.; Gao, Y.; Aerts, M.; Kunneman, L. T.; Schins, J. M.; Savenije, T. J.; van Huis, M. A.; van der Zant, H. S. J.; Houtepen, A. J.; Siebbeles, L. D. A. Unity Quantum Yield of Photogenerated Charges and Band-Like Transport in Quantum-Dot Solids. Nat. Nanotech. 2011, 6, 733-739.

(5) Zheng, Z.; Yim, K.-H.; Saifullah, M. S. M.; Welland, M. E.; Friend, R. H.; Kim, J. S.; Huck, W. T. S. Uniaxial Alignment of Liquid-Crystalline Conjugated Polymers by Nanoconfinement. Nano Lett. 2007, 7, 987-992. 
(6) Geiregat, P.; Justo, Y.; Abe, S.; Flamee, S.; Hens, Z. Giant and Broad-Band Absorption Enhancement in Colloidal Quantum Dot Mono Layers through Dipolar Coupling. ACS Nano 2013, 7, 987-993.

(7) Voros, M.; Galli, G.; Zimanyi, G. T. Colloidal Nanoparticles for Intermediate Band Solar Cells. ACS Nano 2015, 9, 6882-6890.

(8) Kagan, C. R.; Murray, C. B.; Bawendi, M. G. Long-Range Resonance Transfer of Electronic Excitations in Close-Packed CdSe Quantum-Dot Solids. Phys. Rev. B 1996, $54,8633-8643$.

(9) Leatherdale, C. A.; Kagan, C. R.; Morgan, N. Y.; Empedocles, S. A.; Kastner, M. A.; Bawendi, M. G. Photoconductivity in CdSe Quantum Dot Solids. Phys. Rev. B 2000, 62, 2669-2680.

(10) Aerts, M.; Sandeep, C. S. S.; Gao, Y.; Savenije, T. J.; Schins, J. M.; Houtepen, A. J.; Kinge, S.; Siebbeles, L. D. A. Free Charges Produced by Carrier Multiplication in Strongly Coupled PbSe Quantum Dot Films. Nano Lett. 2011, 11, 4485-4489.

(11) Sandeep, C. S. S.; ten Cate, S.; Schins, J. M.; Savenije, T. J.; Liu, Y.; Law, M.; Kinge, S.; Houtepen, A. J.; Siebbeles, L. D. A. High Charge-Carrier Mobility Enables Exploitation of Carrier Multiplication in Quantum-Dot Films. Nat. Commun. 2013, 4, 2360.

(12) ten Cate, S.; Liu, Y.; Sandeep, C. S. S.; Kinge, S.; Houtepen, A. J.; Savenije, T. J.; Schins, J. M.; Law, M.; Siebbeles, L. D. A. Activating Carrier Multiplication in PbSe Quantum Dot Solids by Infilling with Atomic Layer Deposition. J. Phys. Chem. Lett. 2013, 4, 1766-1770.

(13) Rowland, C. E.; Fedin, I.; Zhang, H.; Gray, S. K.; Govorov, A. O.; Talapin, D. V.; Schaller, R. D. Picosecond Energy Transfer and Multiexciton Transfer Outpaces Auger Recombination in Binary CdSe Nanoplatelet Solids. Nat. Mater. 2015, 14, 484-489. 
(14) Sugimoto, H.; Furuta, K.; Fujii, M. Controlling Energy Transfer in Silicon Quantum Dot Assemblies Made from All-Inorganic Colloidal Silicon Quantum Dots. J. Phys. Chem. C 2016, 120, 24469-24475.

(15) Crooker, S. A.; Hollingsworth, J. A.; Tretiak, S.; Klimov, V. I. Spectrally Resolved Dynamics of Energy Transfer in Quantum-Dot Assemblies: Towards Engineered Energy Flows in Artificial Materials. Phys. Rev. Lett. 2002, 89, 186802.

(16) Talapin, D. V.; Murray, C. B. PbSe Nanocrystal Solids for n- and p-Channel Thin Film Field-Effect Transistors. Science 2005, 310, 86-89.

(17) Guyot-Sionnest, P. Electrical Transport in Colloidal Quantum Dot Films. J. Phys. Chem. Lett. 2012, 3, 1169-1175.

(18) Xu, F.; Gerlein, L. F.; Ma, X.; Haughn, C. R.; Doty, M. F.; Cloutier, S. G. Impact of Different Surface Ligands on the Optical Properties of PbS Quantum Dot Solids. Materials 2015, 8, 1858-1870.

(19) Weidman, M. C.; Yager, K. G.; Tisdale, W. A. Interparticle Spacing and Structural Ordering in Superlattice PbS Nanocrystal Solids Undergoing Ligand Exchange. Chem. Mater. 2015, 27, 474-482.

(20) Ross, M. B.; Mirkin, C. A.; Schatz, G. C. Optical Properties of One-, Two-, and Three-Dimensional Arrays of Plasmonic Nanostructures. J. Phys. Chem. C 2016, 120, 816-830.

(21) Duan, C.; Wang, Y.; Sun, J.; Guan, C.; Grunder, S.; Mayor, M.; Peng, L.; Liao, J. Controllability of the Coulomb Charging Energy in Close-Packed Nanoparticle Arrays. Nanoscale 2013, 5, 10258-10266.

(22) Talapin, D. V.; Lee, J.-S.; Kovalenko, M. V.; Shevchenko, E. V. Prospects of Colloidal 
Nanocrystals for Electronic and Optoelectronic Applications. Chem. Rev. 2010, 110, $389-458$.

(23) Yu, D.; Wang, C.; Guyot-Sionnest, P. n-Type Conducting CdSe Nanocrystal Solids. Science 2003, 300, 1277-1280.

(24) Lee, J.-S.; Kovalenko, M. V.; Huang, J.; Chung, D. S.; Talapin, D. V. Band-Like Transport, High Electron Mobility and High Photoconductivity in All-Inorganic Nanocrystal Arrays. Nat. Nanotech. 2011, 6, 348-352.

(25) Jang, J.; Liu, W.; Son, J. S.; Talapin, D. V. Temperature-Dependent Hall and FieldEffect Mobility in Strongly Coupled All-Inorganic Nanocrystal Arrays. Nano Lett. 2014, 14, 653-662.

(26) Ko, D.-K.; Murray, C. B. Probing the Fermi Energy Level and the Density of States Distribution in PbTe Nanocrystal (Quantum Dot) Solids by Temperature-Dependent Thermopower Measurements. ACS Nano 2011, 5, 4810-4817.

(27) Lin, G.; Lu, W. Self-Assembly of Hydrophobic Gold Nanoparticles and Adhesion Property of Their Assembled Monolayer Films. J. Colloid Interface Sci. 2017, 501, 241-247.

(28) Georgiou, G.; Tserkezis, C.; Schaafsma, M. C.; Aizpurua, J.; Gómez Rivas, J. Active Loaded Plasmonic Antennas at Terahertz Frequencies: Optical Control of Their Capacitive-Inductive Coupling. Phys. Rev. B 2015, 91, 125443.

(29) Nijamudheen, A.; Akimov, A. V. Excited-State Dynamics in Two-Dimensional Heterostructures: $\mathrm{SiR} / \mathrm{TiO}_{2}$ and $\mathrm{GeR} / \mathrm{TiO}_{2}(\mathrm{R}=\mathrm{H}, \mathrm{Me})$ as Promising Photocatalysts. $J$. Phys. Chem. C 2017, 121, 6520-6532.

(30) Nijamudheen, A.; Akimov, A. V. Criticality of Symmetry in Rational Design of Chalcogenide Perovskites. J. Phys. Chem. Lett. 2018, 9, 248-257. 
(31) Zaitseva, N.; Dai, Z. R.; Leon, F. R.; Krol, D. Optical Properties of CdSe Superlattices. J. Am. Chem. Soc. 2005, 127, 10221-10226.

(32) Kobayashi, Y.; Nishimura, T.; Yamaguchi, H.; Tamai, N. Effect of Surface Defects on Auger Recombination in Colloidal CdS Quantum Dots. J. Phys. Chem. Lett. 2011, 2, $1051-1055$.

(33) Kim, D.; Tomita, S.; Ohshiro, K.; Watanabe, T.; Sakai, T.; Chang, I.-Y.; HyeonDeuk, K. Evidence of Quantum Resonance in Periodically-Ordered Three-Dimensional Superlattice of CdTe Quantum Dots. Nano Lett. 2015, 15, 4343-4347.

(34) Giansante, C.; Carbone, L.; Giannini, C.; Altamura, D.; Ameer, Z.; Maruccio, G.; Loiudice, A.; Belviso, M. R.; Cozzoli, P. D.; Rizzo, A. et al. Colloidal ArenethiolateCapped PbS Quantum Dots: Optoelectronic Properties, Self-Assembly, and Application in Solution-Cast Photovoltaics. J. Phys. Chem. C 2013, 117, 13305-13317.

(35) Murray, C. B.; Kagan, C. R.; Bawendi, M. G. Synthesis and Characterization of Monodisperse Nanocrystals and Close-Packed Nanocrystal Assemblies. Annu. Rev. Mater. Sci. 2000, 30, 545-610.

(36) Ushakova, E. V.; Cherevkov, S. A.; Litvin, A. P.; Parfenov, P. S.; Volgina, D.-O. A.; Kasatkin, I. A.; Fedorov, A. V.; Baranov, A. V. Ligand-Dependent Morphology and Optical Properties of Lead Sulfide Quantum Dot Superlattices. J. Phys. Chem. C 2016, 120, 25061-25067.

(37) Lingley, Z.; Lu, S.; Madhukar, A. The Dynamics of Energy and Charge Transfer in Lead Sulfide Quantum Dot Solids. J. Appl. Phys. 2014, 115, 084302.

(38) Chistyakov, A. A.; Zvaigzne, M. A.; Nikitenko, V. R.; Tameev, A. R.; Martynov, I. L.; Prezhdo, O. V. Optoelectronic Properties of Semiconductor Quantum Dot Solids for Photovoltaic Applications. J. Phys. Chem. Lett. 2017, 8, 4129-4139. 
(39) Chang, I.-Y.; Kim, D.; Hyeon-Deuk, K. Control of Electronic Structures and Phonon Dynamics in Quantum Dot Superlattices by Manipulation of Interior Nanospace. ACS Appl. Mater. Interfaces 2016, 8, 18321-18327.

(40) Lee, K. D.; Park, M. J.; Kim, D.-Y.; Kim, S. M.; Kang, B.; Kim, S.; Kim, H.; Lee, H.-S.; Kang, Y.; Yoon, S. S. et al. Graphene Quantum Dot Layers with Energy-Down-Shift Effect on Crystalline-Silicon Solar Cells. ACS Appl. Mater. Interfaces 2015, 7, 1904319049.

(41) Carey, G. H.; Yuan, M.; Comin, R.; Voznyy, O.; Sargent, E. H. Cleavable Ligands Enable Uniform Close Packing in Colloidal Quantum Dot Solids. ACS Appl. Mater. Interfaces 2015, 7, 21995-22000.

(42) McGuire, J. A.; Joo, J.; Pietryga, J. M.; Schaller, R. D.; Klimov, V. I. New Aspects of Carrier Multiplication in Semiconductor Nanocrystals. Acc. Chem. Res. 2008, 41, $1810-1819$.

(43) Hyeon-Deuk, K.; Kobayashi, Y.; Tamai, N. Evidence of Phonon-Assisted Auger Recombination and Multiple Exciton Generation in Semiconductor Quantum Dots Revealed by Temperature-Dependent Phonon Dynamics. J. Phys. Chem. Lett. 2014, 5, 99-105.

(44) Govoni, M.; Marri, I.; Ossicini, S. Carrier Multiplication between Interacting Nanocrystals for Fostering Silicon-Based Photovoltaics. Nat. Photon. 2012, 6, 672-679.

(45) Marri, I.; Govoni, M.; Ossicini, S. First-Principles Calculations of Electronic Coupling Effects in Silicon Nanocrystals: Influence on Near Band-Edge States and on Carrier Multiplication Processes. Sol. Energy Mater. Sol. Cells 2016, 145, 162-169.

(46) Marri, I.; Govoni, M.; Ossicini, S. Red-Shifted Carrier Multiplication Energy Threshold and Exciton Recycling Mechanisms in Strongly Interacting Silicon Nanocrystals. J. Am. Chem. Soc. 2014, 136, 13257-13266. 
(47) Kryjevski, A.; Kilin, D. Multiple Exciton Generation in Silicon Quantum Dot Arrays: Density Functional Perturbation Theory Computation. Mol. Phys. 2014, 112, 430-440.

(48) Kryjevski, A.; Kilin, D.; Kilina, S. Amorphous Silicon Nanomaterials: Quantum Dots versus Nanowires. J. Renewable Sustainable Energy 2013, 5, 043120.

(49) Fischer, S. A.; Madrid, A. B.; Isborn, C. M.; Prezhdo, O. V. Multiple Exciton Generation in Small Si Clusters: A High-Level, Ab Initio Study. J. Phys. Chem. Lett. 2010, 1, 232-237.

(50) Bayer, M.; Hawrylak, P.; Hinzer, K.; Fafard, S.; Korkusinski, M.; Wasilewski, Z. R.; Stern, O.; Forchel, A. Coupling and Entangling of Quantum States in Quantum Dot Molecules. Science 2001, 291, 451-453.

(51) Vora, P. M.; Bracker, A. S.; Carter, S. G.; Sweeney, T. M.; Kim, M.; Kim, C. S.; Yang, L.; Brereton, P. G.; Economou, S. E.; Gammon, D. Spin-Cavity Interactions between a Quantum Dot Molecule and a Photonic Crystal Cavity. Nat. Commun. 2015, 6,7665 .

(52) Bracker, A. S.; Scheibner, M.; Doty, M. F.; Stinaff, E. A.; Ponomarev, I. V.; Kim, J. C.; Whitman, L. J.; Reinecke, T. L.; Gammon, D. Engineering Electron and Hole Tunneling with Asymmetric InAs Quantum Dot Molecules. Appl. Phys. Lett. 2006, 89, 233110.

(53) Gawarecki, K.; Machnikowski, P. Phonon-Assisted Relaxation between Hole States in Quantum Dot Molecules. Phys. Rev. B 2012, 85, 041305.

(54) Nikolaev, V. V.; Averkiev, N. S.; Sobolev, M. M.; Gadzhiyev, I. M.; Bakshaev, I. O.; Buyalo, M. S.; Portnoi, E. L. Tunnel Coupling in an Ensemble of Vertically Aligned Quantum Dots at Room Temperature. Phys. Rev. B 2009, 80, 205304.

(55) Chang, I.-Y.; Kim, D.; Hyeon-Deuk, K. Control of Multiple Exciton Generation and 
Electron-Phonon Coupling by Interior Nanospace in Hyperstructured Quantum Dot Superlattice. ACS Appl. Mater. Interfaces 2017, 9, 32080-32088.

(56) Frisch, M. J.; Trucks, G. W.; Schlegel, H. B.; Scuseria, G. E.; Robb, M. A.; Cheeseman, J. R.; Scalmani, G.; Barone, V.; Mennucci, B.; Petersson, G. A. et al. Gaussian09 Revision A.02. Gaussian Inc. Wallingford CT 2009.

(57) Kresse, G.; Furthmüller, J. Efficient Iterative Schemes for Ab Initio Total-Energy Calculations Using a Plane-Wave Basis Set. Phys. Rev. B 1996, 54, 11169-11186.

(58) Ulbricht, R.; Dong, S.; Chang, I.-Y.; Mariserla, B. M. K.; Dani, K. M.; Hyeon-Deuk, K.; Loh, Z.-H. Jahn-Teller-Induced Femtosecond Electronic Depolarization Dynamics of the Nitrogen-Vacancy Defect in Diamond. Nat. Commun. 2016, \%, 13510.

(59) Hyeon-Deuk, K.; Prezhdo, O. V. Multiple Exciton Generation and Recombination Dynamics in Small Si and CdSe Quantum Dots: an Ab Initio Time-Domain Study. ACS Nano 2012, 6, 1239-1250.

(60) Hyeon-Deuk, K.; Prezhdo, O. V. Time-Domain Ab Initio Study of Auger and PhononAssisted Auger Processes in a Semiconductor Quantum Dot. Nano Lett. 2011, 11, $1845-1850$.

(61) Hyeon-Deuk, K.; Prezhdo, O. V. Photoexcited Electron and Hole Dynamics in Semiconductor Quantum Dots: Phonon-Induced Relaxation, Dephasing, Multiple Exciton Generation and Recombination. J. Phys. : Condens. Matter 2012, 24, 363201.

(62) Kobayashi, Y.; Tamai, N. Size-Dependent Multiexciton Spectroscopy and Moderate Temperature Dependence of Biexciton Auger Recombination in Colloidal CdTe Quantum Dots. J. Phys. Chem. C 2010, 114, 17550-17556.

(63) McVey, B. F. P.; Tilley, R. D. Solution Synthesis, Optical Properties, and Bioimaging Applications of Silicon Nanocrystals. Acc. Chem. Res. 2014, 47, 3045-3051. 
(64) Dasog, M.; Bader, K.; Veinot, J. G. C. Influence of Halides on the Optical Properties of Silicon Quantum Dots. Chem. Mater. 2015, 27, 1153-1156.

(65) Wu, C.-L.; Lin, Y.-H.; Cheng, C.-H.; Su, S.-P.; Huang, B.-J.; Chang, J.-H.; Wu, C.-I.; Lee, C.-K.; Lin, G.-R. Enriching Si Quantum Dots in a Si-Rich $\mathrm{SiN}_{\mathrm{x}}$ Matrix for Strong $\chi^{(3)}$ Optical Nonlinearity. J. Mater. Chem. C 2016, 4, 1405-1413.

(66) Das, D.; Samanta, A. Quantum Size Effects on the Optical Properties of nc-Si QDs Embedded in an a-SiO $\mathrm{x}$ Matrix Synthesized by Spontaneous Plasma Processing. Phys. Chem. Chem. Phys. 2015, 17, 5063-5071.

(67) Wu, C.-L.; Su, S.-P.; Lin, G.-R. All-Optical Modulation Based on Silicon Quantum Dot Doped $\mathrm{SiO}_{\mathrm{x}}$ :Si-QD Waveguide. Laser Photonics Rev. 2014, 8, 766-776.

(68) Trinh, M. T.; Limpens, R.; de Boer, W. D. A. M.; Schins, J. M.; Siebbeles, L. D. A.; Gregorkiewicz, T. Direct Generation of Multiple Excitons in Adjacent Silicon Nanocrystals Revealed by Induced Absorption. Nat. Photon. 2012, 6, 316-321.

(69) Robel, I.; Gresback, R.; Kortshagen, U.; Schaller, R. D.; Klimov, V. I. Universal SizeDependent Trend in Auger Recombination in Direct-Gap and Indirect-Gap Semiconductor Nanocrystals. Phys. Rev. Lett. 2009, 102, 177404.

(70) Lifshitz, E. Evidence in Support of Exciton to Ligand Vibrational Coupling in Colloidal Quantum Dots. J. Phys. Chem. Lett. 2015, 6, 4336-4347. 


\section{TOC Graphic.}
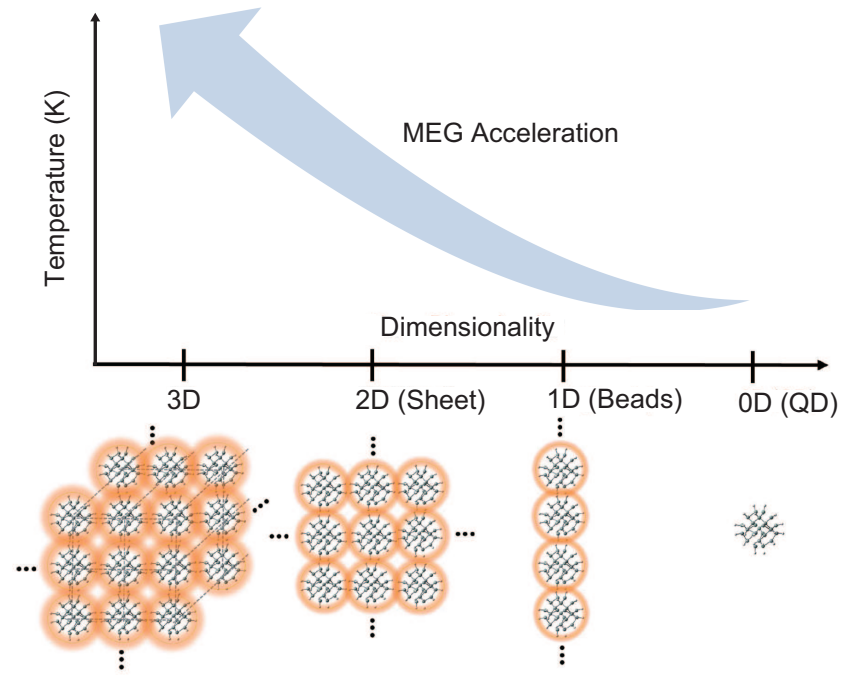


\section{Supporting Information for "Correlated Roles of}

\section{Temperature and Dimensionality for the Multiple}

\section{Exciton Generation and Electronic Structures in}

\section{the Quantum Dot Superlattices"}

I-Ya Chang, ${ }^{\dagger}$ DaeGwi Kim ${ }^{\dagger}$ and Kim Hyeon-Deuk ${ }^{*}, \dagger$

Department of Chemistry, Kyoto University, Kyoto, 606-8502, Japan, and Department of Applied Physics, Osaka City University, Osaka 558-8585, Japan

E-mail: kim@kuchem.kyoto-u.ac.jp

${ }^{*}$ To whom correspondence should be addressed

${ }^{\dagger}$ Department of Chemistry, Kyoto University, Kyoto, 606-8502, Japan

${ }_{\ddagger}^{\ddagger}$ Department of Applied Physics, Osaka City University, Osaka 558-8585, Japan 


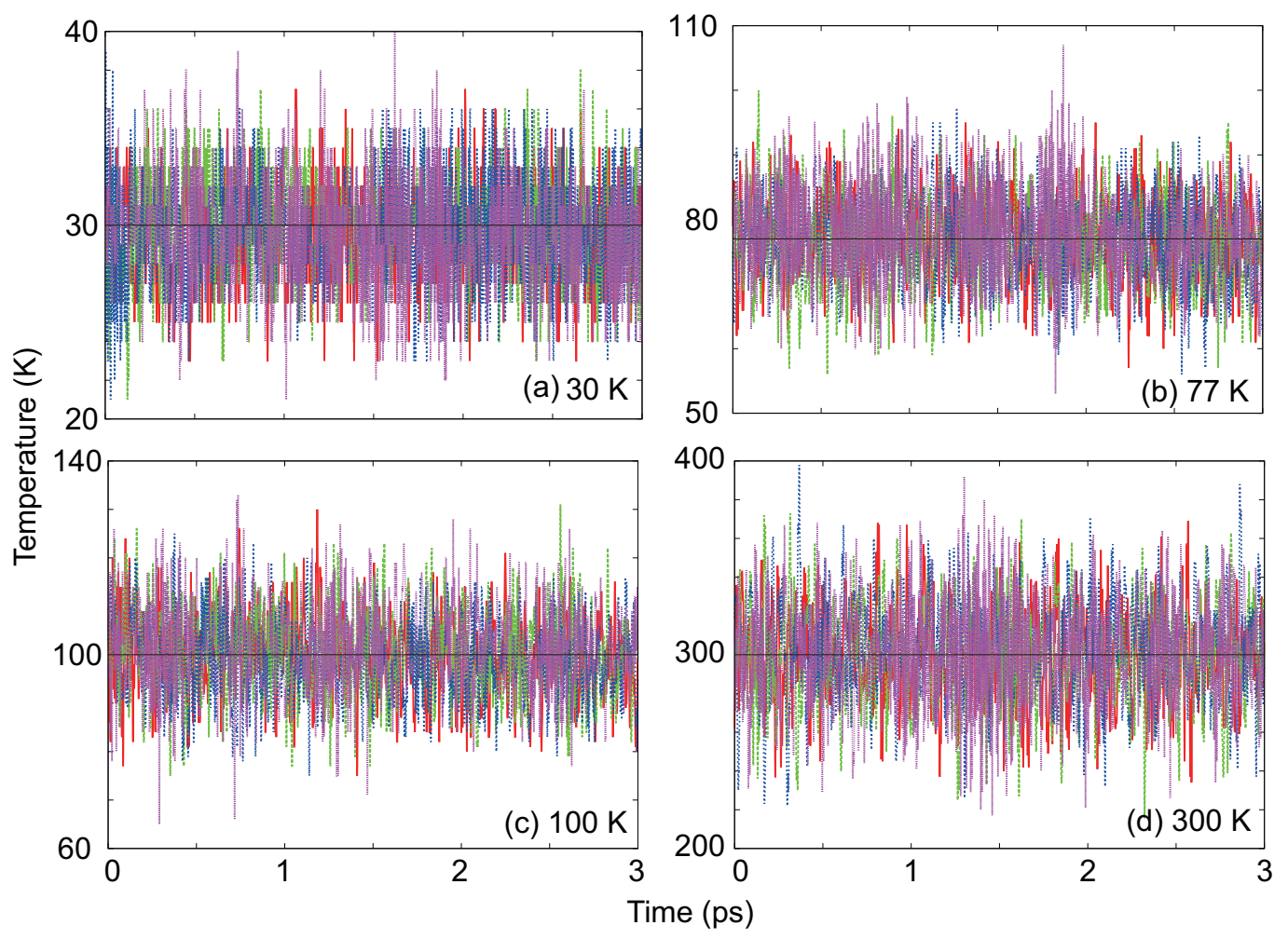

Figure S1: Real-time temperature fluctuations of the $\mathrm{Si}_{29} \mathrm{H}_{24}$ QDSLs and 0D $\mathrm{Si}_{29} \mathrm{H}_{24}$ QD at (a) $30 \mathrm{~K}$, (b) $77 \mathrm{~K}$, (c) $100 \mathrm{~K}$ and (d) $300 \mathrm{~K}$. 

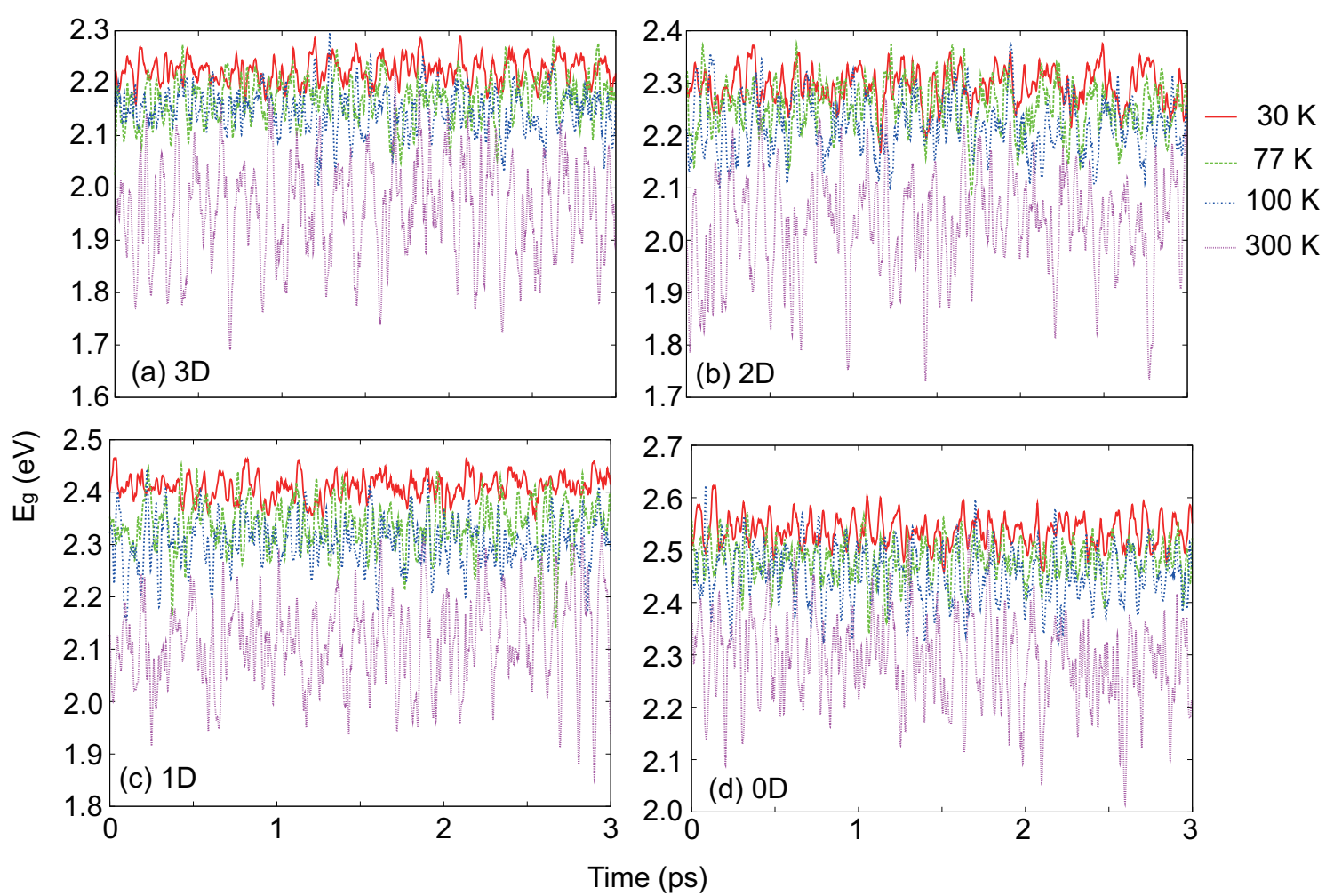

Figure S2: Real-time fluctuations of temperature-dependent band-gap energies of the $\mathrm{Si}_{29} \mathrm{H}_{24}$ QDSLs and 0D $\mathrm{Si}_{29} \mathrm{H}_{24}$ QD. 


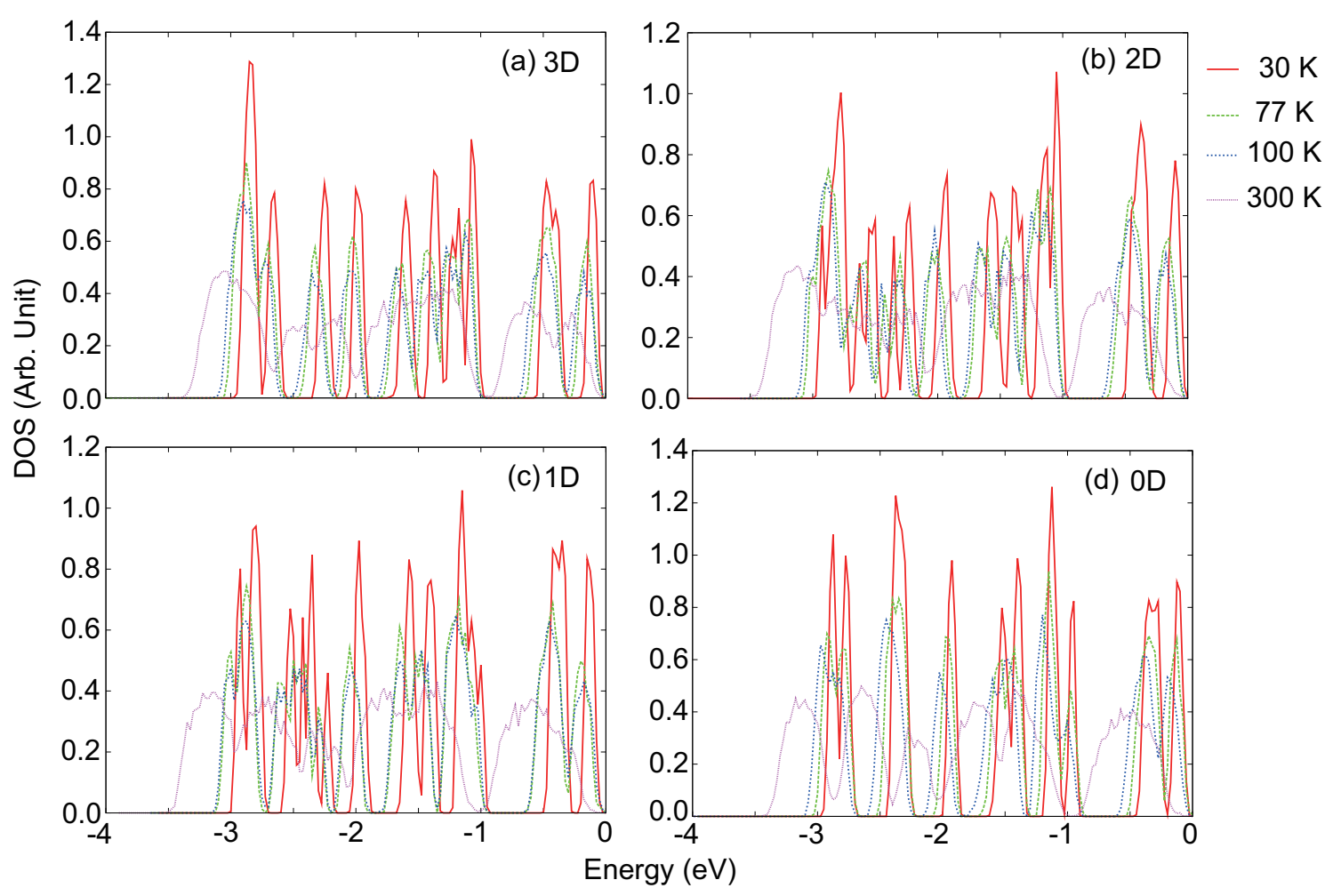

Figure S3: VB DOS for (a) 3D, (b) 2D, and (c) 1D $\mathrm{Si}_{29} \mathrm{H}_{24}$ QDSLs and (d) 0D $\mathrm{Si}_{29} \mathrm{H}_{24}$ QD. The VB DOS was obtained by counting the number of VB states fluctuating at each temperature along the 3 ps microcanonical MD trajectories. Each highest state was shifted to end with $0 \mathrm{eV}$ for their clear comparison. 


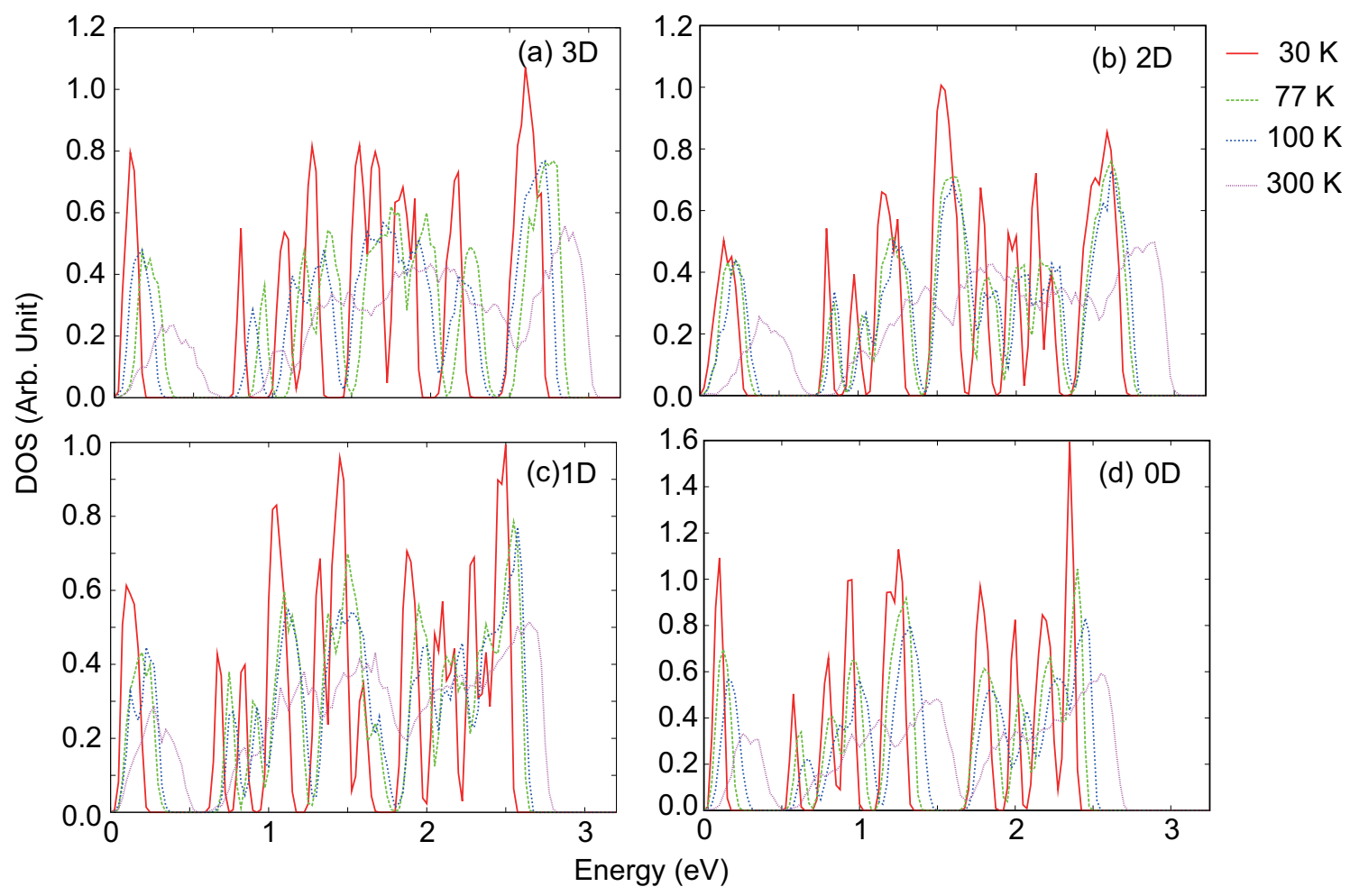

Figure S4: Same as Figure S3 but for the CB states. Each lowest state was shifted to start with $0 \mathrm{eV}$ for their clear comparison. 


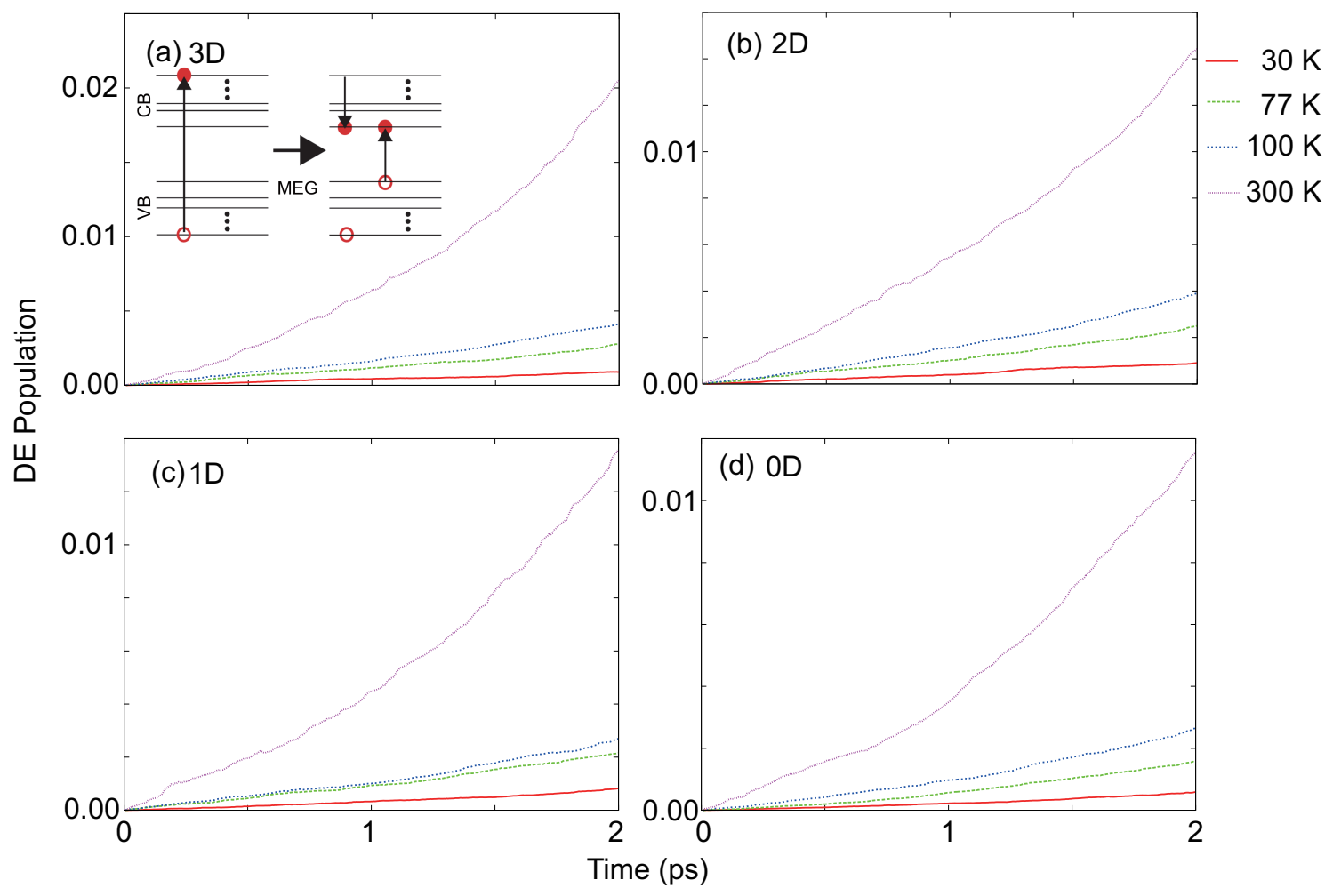

Figure S5: Time-dependent DE population generated through the MEG in (a) 3D, (b) 2D, and (c) $1 \mathrm{D} \mathrm{Si}_{29} \mathrm{H}_{24}$ QDSLs and (d) $0 \mathrm{D} \mathrm{Si}_{29} \mathrm{H}_{24}$ QD. 